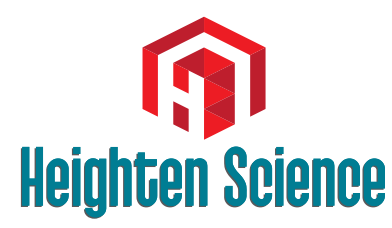

P U B L I C A T I O N S Corporation
*Address for Correspondence: Dr. Fauzia Shafi Khan, Associate Professor, Pediatric Hematology, Children's Hospital/Institute of Child Health, Lahore, Pakistan, Tel: \#00923028488428; Email:

fauzia_khan60@hotmail.com

Submitted: 07 June 2017

Approved: 10 July 2017

Published: 11 July 2017

Copyright: @ 2017 Khan FS, et al. This is an open access article distributed under the Creative Commons Attribution License, which permits unrestricted use, distribution, and reproduction in any medium, provided the original work is properly cited

Check for updates
Research Article

\section{Pure Erythroid Leukemia: The Sole Acute Erythroid Leukemia}

\author{
Fauzia Shafi Khan*, Khalid Mahmood and Alia Ahmad \\ Associate Professor, Pediatric Hematology, Children's Hospital/institute of Child Health, \\ Lahore, Pakistan
}

\section{ABSTRACT}

Pure Erythroid Leukemia (PEL) is an aggressive and exceedingly rare form of acute leukemia. In the 2008 WHO classification PEL was one of the subtypes of acute erythroid leukemia the other subtype being erythroleukemia (erythroid/ myeloid). In the 2016 WHO classification update, erythroleukemia was merged into myelodysplastic syndrome and PEL now is the only type of acute erythroid leukemia. 106 cases of acute myeloid leukemia were diagnosed in 28 months in children's hospital Lahore and PEL constituted $0.94 \%$. Diagnosis of PEL is made by the bone marrow morphology showing predominant Immature erythroid precursors (proerythroblastic or undifferentiated), Periodic Acid-Schiff staining and immunophenotyping. In PEL no specific genetic mutations have been described but complex karyotypes and TP53 mutations are frequently noted. Future collaborative studies to identify the molecular defects will contribute to the development of targeted therapies that might improve the prognosis.

\section{INTRODUCTION}

Acute erythroleukemia (AEL) accounts for 3-5\% of all acute myeloid leukemias (AML) [1]. According to 2008 WHO classification acute erythroid leukemia comprised of pure erythroid leukemia and acute erythroleukemia [2]. In 2016 WHO classification update Pure Erythroid Leukemia (PEL) has been retained as the only type of acute erythroid leukemia, When counting blast percentage the denominator used always is total number of nucleated marrow cells, Percentage of erythroid cells has become irrelevant. PEL is defined as a neoplastic proliferation composed of $>80 \%$ immature erythroid precursors of which proerythroblasts constitute $\geq 30 \%$ [3]. PEL is rare type of acute leukemia patients have a clinically aggressive course. The median survival being only 3 months [4].

\section{AIMS AND OBJECTIVES}

To determine the frequency, clinical presentation, bone marrow morphogy findings and immunophenotype of PEL in children.

\section{METHODS AND INVESTIGATIONS}

We retrospectively evaluated records of all children diagnosed with acute Myeloid Leukemia. In Children's Hospital Lahore between january 2015 to april 2017, total 1120 new cases of acute leukemias were diagnosed and with $109(9.4 \%)$ cases of AML. Two cases of erythroid leukemia diagnosed during this period,theses patients had complete blood counts, reticulocyte count, coombs test, high performance liquid chromatography(HPLC), serum B12, serum folate levels \& underwent bone marrow aspirates. In one case trephine biopsy was done. Immunophenotyping was performed on BD FACS Canto 11, 6 color flow cytometer on bone marrow aspirates. After mononuclear cell enrichment the antibody panel used comprised of B lymphoid 
markers: CD10,CD19,CD20,CD22,cCD79a and T Lymphoid markers CD2, cCD3, CD5, CD7, Myeloid markers CD13, CD33, CD117, MP0, Monocytic markers: CD11c, CD14, Erythroid markers: Anti glycophorin A, Megakaryoblastic markers: CD61, Others: HLADR, Anti Tdt,CD34.

\section{RESULTS}

Different FAB subtypes of AML diagnosed (Table 1). Average age of AML children was 7.6 years \& ranged from 2 months to 16 years,there were 67 males \& 39 females, M:F being 1.7:1.

Patients diagnosed with M6 were both male, The first was 8yrs old, immunophenotyping of bone marrow aspirate showed CD13+, CD34+, CD117+, HLADR+ and dim (MPO+\& CD33+) in myeloid blast population (20\%) \& Anti Glycophorin A positivity \& HLADR negativity in erythroid population that had dim CD45+, this patients was diagnosed as Acute Erythroid Leukemia in 2015 and would now not be classified as such according to WHO 2016 classification.

The other patient was a 14 months old male, presented with pallor, fever \&abdominal distention. Hematological investigation revealed hemoglobin of $61 \mathrm{~g} / \mathrm{L}$ ), Leukocytes were $4 \times 10^{9} / \mathrm{L}$, platelet count was $88 \times 10^{9} / \mathrm{L}$. Coombs test was negative, HPLC, Serum B12 and serum folate levels were within normal limits. Bone marrow aspirate (Giemsa's stain) was hypercellular predominantly composed of erythroid precursors (85\%) with significant dysplasia including nuclear budding, multinucleation and megaloblatoid change, proerythroblasts constituted $43 \%$ of the erythroid component while myeloid and megakaryocytic morphology was normal. Periodic Acid Schiff (PAS) stain revealed coarse granular and block positivity in many of the erythroblasts (Figure 1).

\begin{tabular}{|c|c|}
\hline Table 1: Number of patients of different FAB AML subtypes diagnosed. \\
\hline AML MO & 04 \\
\hline AML M1 & 13 \\
\hline AML M2 & 38 \\
\hline AML M3 & 16 \\
\hline AML M4 & 18 \\
\hline AML M5a & 09 \\
\hline AML M5b & 04 \\
\hline AML M6 & 02 \\
\hline AML M7 & 02 \\
\hline TOTAL & 106 \\
\hline
\end{tabular}

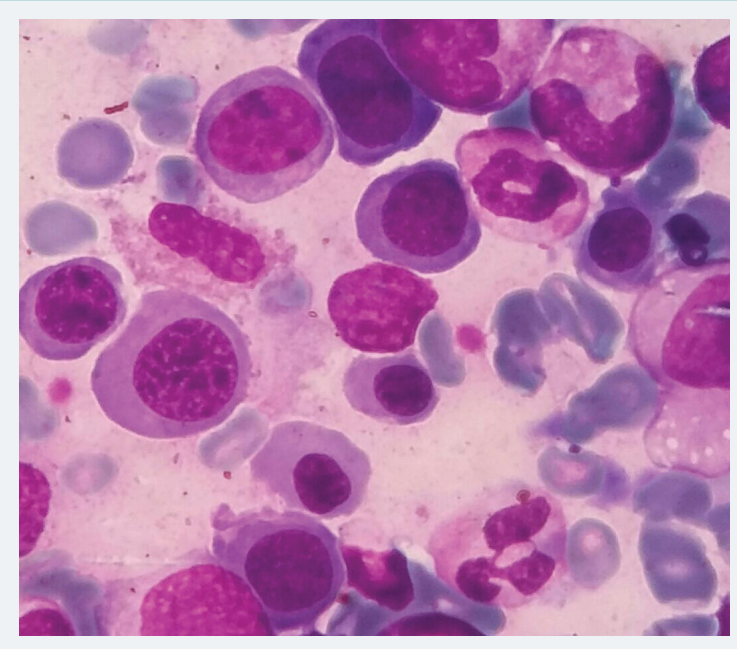

Figure 1: Bone marrow aspirate showing markedly dysplastic erythroid precursors (Giemsa's Stain 1000x). 
On bone marrow aspirate immunophenotyping erythroid population comprised $82 \%$ of nucleated marrow cells with dimCD45+, anti Glycophorin A positivity \& negative for myeloid markers (MPO,CD13,CD33,CD61), B and T cell markers (CD10, CD19, cCD79a, HLADR, Anti Tdt, CD2, cCD3, CD5), monocytic markers CD11c CD14 Megakaryoblastic markers: CD61, Others: CD34, anti-kappa, anti-lambda, CD45.

\section{DISCUSSION}

Pure erythroid leukemia is a very rare type of AML it represents $<1 \%$ of all AML cases and $13 \%$ of acute erythroid leukemia cases [4]. In our study it comprised $0.94 \%$ of total AML cases. PEL is morphologically and immunophenotypically distinct from other cases of AML with erythroid hyperplasia [5].

In PEL there is neoplastic proliferation of immature cells committed solely to an erythroid lineage with no increase in myeloblasts in the bone marrow [6-8]. Patients usually present with profound anemia hemorrhages and fever [9]. In $20-40 \%$ cases hepatosplenomegaly can be present $[9,10]$, our patient also had similar presentation.

Peripheral blood smears shows nonspecific changes in the majority of cases, circulating erythroblasts may be seen in about a quarter of patients [6]. Anemia and thrombocytopenia are commonly observed [4], our patient had pancytopenia and died before treatment. In bone marrow aspirate, the predominant cells $(>80 \%)$ are erythroid precursors with left shift and increased proerythroblasts which are the most immature recognizable erythroid precursor. they are medium to large blasts with round nuclei, fine nuclear chromatin, occasional prominent nucleoli,cytoplasm is deep blue and is agranular with variable cytoplasmic vacuolization [11], in our patient the marrow aspirate was also hypercellular with erythroid component constituting $85 \%$ with left shifted maturation and pronormoblasts comprising $43 \%$ of the erythroid component and marked dyserythropoiesis. Dyserythropoiesis is prominent, while dysplasia in myeloid and megakaryocytic lineages is not an obvious feature [12]. Rarely the blasts are smaller and resemble lymphoblasts of Acute Lymphoblastic Leukemia [13].

Periodic Acid-Schiff (PAS) cytochemical staining is commonly positive, in a blocklike pattern, sudan black MPO, and chloroacetate esterase are characteristically negative [14]. Bone marrow biopsy biopsy is usually hypercellular and shows erythroid predominance with sheets of immature cells [4], bone marrow trephine biopsy and cytogenetics were not performed on our patient and the patient died before.

Immunophenotyping on bone marrow aspirate of our patient the erythroid population comprised $82 \%$ of nucleated marrow cells with dim CD $45+$, anti Glycophorin A positivity \& negative for myeloid, lymphoid, monocytic and megakaryocytic markers.

Glycophorin A positivity has been reported to be consistent with erythroid precursors, CD71 \& partial CD36 positivity also seen in erythroid precursors [15]. Pure erythroid leukemia without morphologic evidence of erythroid maturation may be difficult to be distinguished from other types of AML, particularly megakaryoblastic leukemia, and also from acute lymphoblastic leukemia and lymphoma, E-cadherin a cell adhesion molecule is a selective marker of immature erythroblasts and is down regulated during erythroid maturation. In the bone marrow, its expression appears mainly in immature erythroblasts [16-18], beta-sialoglycoprotein, carbonic anhydrase1, can also be used to identify minimally differentiated M6 [19]. Immature erythroid precursors comprise the predominant component of PEL, using markers that specifically determine erythroid lineage is crucial. Ferritin is only positive in early erythroid precursors, whereas more mature forms tend to be weak or negative, thus, it is another specific marker of early erythroid precursors in PEL [20]. 
Non-neoplastic conditions included in PEL differential diagnosis include congenital dyserythropoietic anemia, hemolytic anemia, vitamin B12/folate deficiency and infection [21] and Neoplastic conditions included are myeloid neoplasms with erythroid predominance like MDS, acute myeloid leukemia with myelodysplasia related changes(AML-MRC), AML with recurrent cytogenetic abnormalities and therapy related AML [22].

PEL is a very aggressive and rare disease the standard induction produces complete remission (CR) in only $10-40 \%$ cases, which is usually brief. No specific genetic mutations have been described in PEL, mutations found in other types of AML types, such as FLT3, NPM1, and CEBPA, are very rarely present in PEL It frequently presents with a complex karyotype and TP53 mutations. The molecular mechanisms of PEL are unknown, there is no targeted therapy and thus the dismal prognosis. Future collaborative studies to identify the molecular defects will contribute to the development of targeted therapies which might improve its outcome.

\section{REFERENCES}

1. Santos FP, Bueso-Ramos CE, Ravandi F. Acute erythroleukemia: Diagnosis and management. Expert Rev Hematol. 2010; 3: 705-718. Ref.: https://goo.gl/6kD1fj

2. Swerdlow SH, Campo E, Harris NL. WHO Classification of Tumours of Haematopoietic and Lymphoid Tissues. Lyon, France. IARC Press. 2008.

3. Arber DA, Orazi A, Hasserjian R, Thiele J, Borowitz MJ, et al. The 2016 revision to the World Health Organization classification of myeloid neoplasms and acute leukemia. Blood. 2016; 127: 2391-2405. Ref.: https://goo.gl/2XHvJ2

4. Liu W, Hasserjian RP, Hu Y, Zhang L, Miranda RN, et al. Pure erythroid leukemia: a reassessment of the entity using the 2008 World Health Organization classification. Mod Pathol. 2011; 24: 375-383. Ref.: https://goo.gl/rM35Zr

5. Wong E, Ling V, Westerman D, Morgan S, Juneja S. How unique is pure erythroid leukaemia? A retrospective analysis of seven cases and review of the literature. J Clin Pathol. 2015; 68: 30-35. Ref.: https://goo.gl/M7uYcW

6. Wang SA, Hasserjian RP. Erythroid proliferations in myeloid neoplasms. Hum Pathol. 2012; 43: 153164. Ref.: https://goo.gl/i9SJFC

7. Liu W, Hasserjian RP, Hu Y, Zhang L, Miranda RN, et al. Pure erythroid leukemia: a reassessment of the entity using the 2008 World Health Organization classification. Mod Pathol. 2011; 24: 375-383. Ref.: https://goo.gl/rM35Zr

8. Liu CJ, Hong YC, Yang CF, Liu SH, Gau JP, et al. Clinicopathologic features and outcome of acute erythroid leukemia based on 2008 revised World Health Organization classification. Leuk Lymphoma. 2012; 53: 289-294. Ref.: https://goo.gl/jVuATn

9. Olopade OI, Thangavelu M, Larson RA, MickR, Kowal-Vern A, et al. Clinical, morphologic, and cytogenetic characteristics of 26 patients with acute erythroblastic leukemia. Blood. 1992; 80: 2873-2882. Ref.: https://goo.gl/KvLhye

10. Colita A, Belhabri A, Chelghoum Y, Charrin C, Fiere D, et al. Prognostic factors and treatment effects on survival in acute myeloid leukemia of M6 subtype: a retrospective study of 54 cases. Ann Oncol. 2001; 2: 451-455. Ref.: https://goo.gl/MFG7i1

11. Hasserjian RP, Zuo Z, Garcia C, Tang G, Kasyan A, et al. Acute erythroid leukemia: a reassessment using criteria refined in the 2008 WHO classification. Blood. 2010; 115: 1985-1992. Ref.: https://goo.gl/VrAZBp

12. Mihova D, Zhang L. Acute erythroid leukemia: A review. N A J Med Sci. 2012; 5: 110-118.

13. Swerdlow SH, Campo E, Harris NL. WHOclassification of tumors of haematopoietic and lymphoid tissues (IARC WHO classification of tumours), 4th edn. WHO Press, Lyon. 2008.

14. Zuo Z, Polski JM, Kasyan A, Medeiros LJ. Acute erythroid leukemia. Arch Pathol Lab Med. 2010; 134: 1261-1270. Ref.: https://goo.gl/7WUxno

15. Aljabry M. Complex Karyotype with Novel Translocation in Pure Erythroid Leukemia Patient. Hematol Rep. 2015; 7: 5674. Ref.: https://goo.gl/Xe6h3Mb

16. Acs G, LiVolsi VA. Loss of membrane expression of E-cadherin in leukemic erythroblasts. Arch Pathol Lab Med. 2001; 125: 198-201. Ref.: https://goo.gl/Rd75Nj 
17. Armeanu S, Bühring HJ, Reuss-Borst M, Müller CA, Klein G. E-cadherin is functionally involved in the maturation of the erythroid lineage. J Cell Biol. 1995; 131: 243-249. Ref.: https://goo.gl/qu62Xv

18. Bühring HJ, Müller T, Herbst R, Cole S, Rappold I, et al. The adhesion molecule E-cadherin and a surface antigen recognized by the antibody $9 \mathrm{C} 4$ are selectively expressed on erythroid cells of defined maturational stages. Leukemia. 1996; 10: 106-116. Ref.: https://goo.gl/hAVkdm

19. Southcott MJ, Tanner MJ, Anstee DJ. The expression of human blood group antigens during erythropoiesis in a cell culture system. Blood. 1999; 93: 4425-4435. Ref.: https://goo.gl/qquCUH

20. Wang W, Grier DD, Woo J, Ward M, Sui G, et al. Ferritin $\mathrm{H}$ is a novel marker of early erythroid precursors and macrophages. Histopathology. 2013; 62: 931-940. Ref.: https://goo.gl/6SwjTQ

21. Moharram L, Kamal N, Sukhun SA, Sughayer MA. Erythropoietin-induced acute erythroid leukemia-like picture: a potential pitfall. Hematol Oncol Stem Cell Ther. 2014; 7: 50-52. Ref.: https://goo.gl/PwV9ZY

22. Arber DA, Brunning RD, Orazi A. Acute myeloid leukemia, not otherwise specified. Swerdlow $S H$, Campo E, Harris NL, et al. editors. Tumours of haematopoietic and lymphoid tissues. Lyon: IARC Press. 2008; 134-136. 